\title{
THE SPECIAL VULNERABILITY OF THE MACULAR FIBRES AND “SPARING OF THE MACULA"
}

BY

\author{
H. M. TRaQuair
}

EDINBURGH

IT is widely believed amongst ophthalmologists that the macular fibres in the visual path, especially in the optic nerve, are much more liable to disease than those which correspond to the retinal periphery, and also, though doubtless with fewer adherents and somewhat more reserve, that each macula is connected with both cortical visual centres so that when one is destroyed full central vision may still be preserved. The former hypothesis is based upon the study of the symptoms of retrobulbar neuritis and toxic amblyopia in which central scotoma is a characteristic feature, the latter on the study of occipital hemianopias of vascular origin in which " sparing" of the fixation point is the rule.

It is not easy to see why there should be special vulnerability below the geniculate ganglion and special immunity above it, and it may be of interest to review the subject in the light of our present knowledge.

With regard to the retina it may be concluded that the macular area, being constantly directed towards illuminated objects, is more exposed to light and to variations in light than other parts of the retina and that, therefore, metabolism is more active and more constant in this area. On these grounds the macula might be expected to show signs of wear at a relatively early stage. That some cases of macular disease result from causes of this kind may not be impossible, but if such causes were of any importance the incidence of macular disease amongst those whose work requires the constant use of closely applied central vision would surely be greater than it is. It can hardly be denied, however, that certain forms of disease of the central visual elements appear to support the hypothesis of special vulnerability, but when we examine the evidence closely it becomes clear that while the central elements as a whole are easily affected it is by no means always those whose physiological function is most highly elaborated which suffer soonest or most severely.

Frequently the paracentral or parafoveal elements are mainly involved. In many cases of central choroido-retinal disease the ophthalmoscopic appearances suggest gross and severe lesions and yet central vision remains relatively good. If the scotoma in such cases is carefully analyzed, it will often be found that small areas of practically absolute blindness are situated here and there 
around the fixation point, perhaps partly invading it, but that central vision itself may remain but little affected for long after paracentral vision has failed in patches. Not infrequently a definite small ring scotoma can be discovered in such cases, encircling an area of comparatively good vision. Ring scotoma occurs in both retinal and optic nerve disease and the central seeing area may be quite small.

The occurrence of ring scotoma in choroido-retinal and nerve disease suggests that the incidence of the injury to the nerve elements is determined by causes quite other than functional activity, and the same may be said in respect of conditions characterized by early peripheral visual loss such as tabetic and post-oedematous atrophy, retinitis pigmentosa, glaucoma, and others. With regard to the effects of toxins the different results produced by different poisons seem incapable of any other explanation than that of selective affinity. Some toxic substances cause defects chiefly in the central parts of the field, others in the periphery, and in the former group there seems to be little or no special predilection for the exact central elements whether cells or fibres. This is most clearly demonstrable in the case of tobacco poisoning in which the slow development of the visual interference enables its primary incidence and progress to be accurately ascertained. The nerve elements related to the area between the papilla and the macula seem to be very susceptible, and the common and important centro-caecal type of scotoma is expressive of this weakness. This defect may occur in tobacco poisoning and in retrobulbar neuritis from various causes. In tobacco poisoning the intense part of the defect is never central through in advanced cases the fixation point may become for a time overlapped by the edge of the area of most intense blindness. In various forms of retrobulbar neuritis with a centro-caecal defect, the conditions are somewhat similar though here the foveal area often suffers more severely and the scotoma may be large and dense throughout. Moreover, in bilateral cases the defects are sometimes different on the two sides, being chiefly central in one field and peripheral in the other.

In the more acute forms of central toxic defect the incidence is not yet definitely known, and until evidence can be obtained from the examination of the earliest stages of such conditions we are not entitled to believe that the susceptibility of the nerve elements is governed by their function. Again, the observation of central defects during recovery frequently shows that the fixation area is not the last part to be restored. Paracentral defects of considerable intensity may remain after the restitution of normal central vision. In retrobulbar neuritis it may sometimes be observed that, after a period of slow and slight improvement, a 
rapid access of central vision occurs. If the field be tested at this time the explanation will often be found in the presence of a ring scotoma, central vision having emerged through the former large scotoma as a mountain peak protrudes through mist. The recovery of central vision may be very slow, it may occur after a year or more, a result hardly to be expected if the central elements were anything but highly resistant. As the visual path passes centralwards the conditions under which visual loss occurs change. In the optic nerve retrobulbar neuritis of various kinds and damage caused by endogenic and exogenic toxins are the usual causes of conduction interference, pressure and vascular disease being comparatively uncommon. It is of interest to note that the condition or group of conditions included under the term retrobulbar neuritis, which is such a frequent cause of central scotoma, is almost confined to that part of the nerve path which is provided with a skeleton of fibrous trabeculae, i.e., the optic nerve. In the chiasma and tracts the direct action of toxins almost disappears and the liability to disease is accordingly considerably less than in the subchiasmal path. Tumour pressure, acting through the medium of the circulation, syphilis and multiple sclerosis are the chief causes of chiasmal or tract interference. At this level lesions produce hemianopia and the dividing line between the seeing and the defective half fields passes either vertically through the fixation area or skirts round it into the defective half field. In the latter case there is said to be "sparing of the macula," more correctly, sparing of the fixation area. In chiasmal and tract lesions sparing of the fixation area is frequent though not so common as in suprageniculate lesions. The sparing may be small in amount and relative, i.e., present for large objects and absent for colours or small stimuli, and the amount of sparing may vary from time to time or differ on the two sides. In certain tumour cases where the sparing was absent it has been found to appear after relief of pressure by operation. In complete blocking or destruction of the tract there is no sparing. In the suprageniculate part of the pathway the nature of the cause of visual loss changes again. Vascular lesions resulting in diminution or cessation of blood supply become prominent, also pressure from tumours, abscesses, or haemorrhage near though not involving the visual elements. Direct toxic interference or anything comparable to the "neuritis" of the subchiasmal path does not appear to occur. Sparing of the fixation area is the rule. Only in wounds, in which actual destruction by trauma of the macular cortical centre or its fibres can take place, is division of the fixation area at all common. In certain vascular lesions, however, the macular area may be divided and in the case of tumours which gradually compress the radiations the fixation area may be spared at first 
and' divided later. The latter observation forms the basis of the view advocated by Lenz that at a point in the optic radiation near the boundary between the parietal and occipital lobes a bifurcation of the central fibres takes place so that both sides of each macular area are connected with each occipital centre. Interference in front of this point is likely to involve the whole radiation and Lenz has shown that division of the fixation area is more common in anterior hemianopias.

In occipital vascular lesions sparing of the fixation area is the rule at the time when the field is examined but it is sometimes absent or only relative, while sometimes it is absent at first and appears later. It is probable that, if every case of vascular occipital hemianopia could be examined at the earliest moment that perimetry is possible, division of the fixation point would be more frequently found.

Lastly, we may note that hemianopic central scotomata may occur due to occipital lesions of vascular type; here the fixation area is divided and recovery does not occur. These scotomata are similar to those produced by wounds and are evidently due to a direct lesion of the macular elements.

If we review the genesis of central scotomata due to nerve lesions it becomes evident that they are much more likely to be produced by lesions of the anterior than of the posterior part of the visual path, while sparing of the fixation area becomes more common the more posteriorly the lesion is situated. We have also seen that the nature of the lesion varies with the part of the path affected.

Involvement of the central fibres is common where the nerve path is concentrated and more infrequent where the fibres are spread out and in no case is there evidence that those elements which are hardest worked or functionally most highly evolved are specially liable to suffer.

In the subgeniculate pathway it is of interest to note that the acuity of the lesion appears to predispose to central interference. This is most noticeable in the case of pressure interference with the chiasma. Slowly growing or stationary pituitary tumours, as in chronic acromegaly, are associated with bitemporal hemianopic defects without central scotomata, whereas actively growing tumours or inflammàtory processes practically always produce pronounced central defects. In the optic nerve this feature is less marked, but while all the acute changes are characterized by central scotoma, most of the more chronic processes, such as slowly advancing tabetic atrophy and post-neuritic atrophy from intracranial pressure, are distinguished by its absence.

The most satisfactory explanation of sparing of the fixation area in occipital vascular lesions is that advocated by Lister and 
Holmes, i.e., that it is due to survival of the cortical macular area owing to its potential double blood supply from the middle as well as from the posterior cerebral artery. Henschen has also pointed out that the optic radiation in its posterior part receives deep twigs from the middle cerebral artery. The terminal branches of these arteries anastomose freely in the pia mater, but the penetrating twigs are end arteries. In this way initial division of the fixation area followed by sparing is easily accounted for, and isolated homonymous hemianopic scotomata may be explained on the assumption that the block has occurred in one or several penetrating twigs distal to the pial anastomosis.

The functional superiority of the macular neurones also suggests that a greater degree of interference may be required to put them completely out of action, whether it is caused by vascular disease or by pressure on the radiation by a tumour. For these reasons it seems unnecessary to postulate a double cortical representation for the macular area although this hypothesis is advocated by such recognized authorities as Wilbrand and Saenger. As regards central scotomata it would appear that the macular cells and fibres are in reality extremely resistant, and that their liability to interference depends upon anatomical and circulatory conditions and on selective affinities for toxins rather than upon functional relationships.

\title{
A SERIES OF 250 CATARACT EXTRACTIONS BY BARRAQUER'S METHOD*
}

BY

\author{
MAJOR R. E. WRIGHT, I.M.S.
}

SUPERINTENDENT, GOVERNMENT OPHTHALMIC HOSPITAL, MADRAS

THE method of removing the cataractous lens in its capsule by means of a suction apparatus, as perfected by Barraquer, attracted a large amount of attention throughout the ophthalmological world shortly after it was described and demonstrated. As in numerous other clinics the method was put on its trial here as soon as practicable.

In 1922-23 a series of 250 cases was completed. The operations did not run consecutively but parallel with the routine "Madras" operation (primary capsule rupture with complete iridectomy) and

* From material collected for and included in the Annual Report of the Superintendent, Government Ophthalmic Hospital, Madras for 1922. 